\section{§13. Experimental Research on Molten Salt Thermofluid Technology Using a High-Temperature Molten Salt Loop Applied for a Fusion Reactor Flibe Blanket}

Toda, S., Yuki, K.,

Chiba, S., Omae, M. (Tohoku University), Sagara, A.

It was shown in the last report that molten-salt flow had to be stirred by different ways from a mixing method applied for usual fluids like water due to its high viscosity, and that a packed-bed tube was effective to stir the flow in order to obtain accurate bulk temperatures.

However, as this experiment is performed under high-temperature and high Pr-number conditions, we also have to consider the suitable shape of the test section containing the mixing chamber in which the bulk temperature are measured. The 1st test section, which we had constructed first, had too high heat capacity because of the complicated mixing chamber, so that it was difficult to measure heat transfer coefficients. On the other hand, it is possible to obtain accurate results compared with an experimental formula as shown in Fig. 1 because the 2 nd test section has smaller heat capacity and a compactness.

Furthermore, it is clarified that the molten-salt flow through the mixing pipe with the inner diameter of $\phi=19 \mathrm{~mm}$ is well stirred with packed beds $(\phi=9 \mathrm{~mm})$ especially at high flow rate, though the flow is not stirred enough at low flow rate (Fig. 2). From this result, we have to investigate the mixing chamber design for high Pr number fluid.

The experimental plannings with this 2nd test section are as follows; at first, we will have to estimate the heat transfer coefficient under higher temperature conditions through a smooth pipe and to compare the data with an experimental formula. Secondary, we should find out the most suitable method for the fluid mixing by estimating the effects of the length of the mixing area and the size of the packed bed particles. Thereafter, we will investigate heat transfer enhancements applying the packed-bed tubes, twisted-tape inserted tubes and another possible turbulence promoter tubes.

Also, it was obtained to handle the high-temperature molten salt loop. In operating this loop, the molten salt has to be melted in a Dump Tank at first because the salt is stored at a solid state ordinarily. As a heater for the Dump Tank is being installed around its outside wall, melting of the salt proceeds like a "whiskey on the rock." The salt starts to melt from the region adjacent to the wall and pipes, which makes "a cracking sound."

There won't be any problems only with this sound. However, the melting process makes a extraordinary big sound with "a shock," that is heard once whenever the salt melts. The inside of the Dump Tank cannot be confirmed visibly, it may be caused by buoyancy of the molten salt or a difference of thermal expansion between the SUS316 pipes and the molten salt. As this shock may destroy the Dump Tank, we have to grasp this phenomenon.

This phenomenon is important for handling this loop. And a number of other phenomena that will be obtained from future operation will make it clearer to handle the high-temperature molten salt loop.

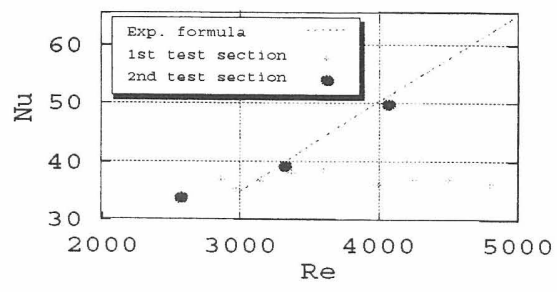

Figure 1: Re vs $\mathrm{Nu}$

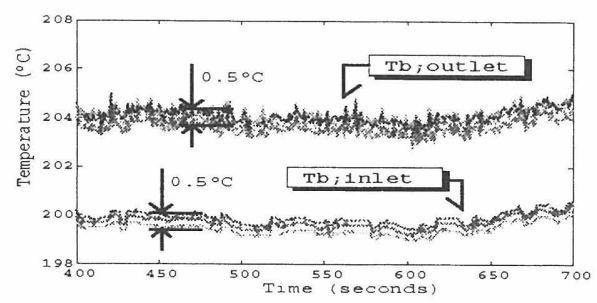

(a) High flow rate

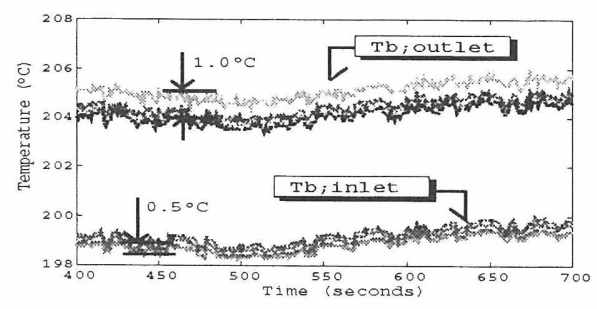

(b) Low flow rate

Figure 2: Bulk Temperature at inlet and outlet of heating area 